\title{
Introducing a re-sampling methodology for the estimation of empirical macroscopic fundamental diagrams
}

\section{Working Paper}

Author(s):

Ambühl, Lukas; Loder, Allister; Bliemer, Michiel; Menendez, Monica; Axhausen, Kay W. (D)

Publication date:

2017-08

Permanent link:

https://doi.org/10.3929/ethz-b-000175247

Rights / license:

$\underline{\text { In Copyright - Non-Commercial Use Permitted }}$

Originally published in:

Arbeitsberichte Verkehrs- und Raumplanung 1271 
1 Introducing a re-sampling methodology for the estimation of empirical macroscopic

2 fundamental diagrams

3 Date of submission: 2017-08-1

Lukas Ambühl

IVT, ETH Zürich,

CH 8093 Zürich, Switzerland

phone: +4144633 3251

fax: +41446331057

lukas.ambuehl@ivt.baug.ethz.ch

\author{
Allister Loder \\ IVT, ETH Zürich, \\ CH 8093 Zürich, Switzerland \\ phone: +414463362 58 \\ fax: +41446331057 \\ allister.loder@ivt.baug.ethz.ch
}

\title{
Michiel C.J. Bliemer
}

Institute of Transport and Logistics Studies,

University of Sydney,

NSW 2006, Australia

phone: +61291141840

fax: +61291141863

michiel.bliemer@sydney.edu.au

10

Monica Menendez

IVT, ETH Zürich, Switzerland,

CH 8093 Zürich

phone: +41446336695

fax: +41446331057

menendez@ivt.baug.ethz.ch

12

Kay W. Axhausen

IVT, ETH Zürich,

CH 8093 Zürich, Switzerland

phone: +41 446333943

fax: +41 446331057

axhausen@ivt.baug.ethz.ch

${ }_{14}$ Words: 5352 words +4 figures +1 table $=6602$ word equivalents 


\begin{abstract}
The uncertainty in the estimation of the macroscopic fundamental diagram (MFD) under realworld traffic conditions and urban dynamics, might result in an inaccurate estimation of the MFD parameters - especially if congestion is rarely observed network-wide. For example, if the observed data does not exhibit a distinct congested branch, it is hard to determine the network capacity and critical density. Similarly, as the data normally comes from punctual observations out of the whole network, it is unclear how representative these observations might be (i.e. how much is the observed capacity affected by the network's inhomogeneity). This, in turn, also leads to uncertainties and errors in the parametrization of the MFD for applications, e.g. traffic control.

In this paper we introduce a novel methodology to estimate (i) the critical density of the MFD, even when no congested branch is observed, and (ii) the level of inhomogeneity in the network. The methodology is based on the idea of re-sampling the empirical data set. Using an extensive data set from Lucerne, Switzerland, and London, UK, we provide insights on the performance and the application of the proposed methodology. We show that, for the network of Lucerne, the proposed methodology allows us to accurately estimate the critical density up to 16 times more often than it would be possible otherwise. This simple and robust estimation of the critical density is crucial for the application of many traffic control algorithms. Additionally, we also use the proposed methodology to illustrate how the level of inhomogeneity is lower in Lucerne than in the three areas of the network of London that we investigate. The proposed measure of the level of inhomogeneity gives city planners the possibility to analyze and investigate how efficiently their road network is utilized.
\end{abstract}




\section{INTRODUCTION}

2 The macroscopic fundamental diagram (MFD) is the upper bound in the macroscopic flowdensity $(q-k)$ relationship for vehicular traffic in an urban road network. Similar to a fundamental diagram of a single road, MFDs are characterized by an uncongested branch and a congested branch. In the uncongested branch, flow increases with density, whereas in the congested branch, flow decreases with increasing density until gridlock is reached, and speeds drop to zero. Thus, MFDs can be described by a combination of some of the following parameters: free flow speed, backward wave speed, capacity, jam density, critical density, and a smoothing parameter that describes the change in the slope of the average speed. Notice that for control purposes, the critical density is an important parameter, as it marks the difference between the congested and the uncongested branch. The shape of the MFD is determined by the urban road structure and the traffic control (1), but more or less independent of the demand (assuming trip length remains more or less constant). New applications have been developed exploiting the elegance of the MFD to model traffic dynamics, predict travel behavior, and control traffic in urban networks (29), all relying on an accurate estimation of the MFD.

The MFD can be either analytically derived or approximated from empirical data (10). Daganzo and Geroliminis (1) proposed a semi-analytical formulation for urban networks. However, their method relies on technical information (green times, number of links that can be passed by a fast vehicle without stopping, etc.), which might be highly variant or not available at all. The MFD can also be directly estimated from empirical data leading to a concave and to some extent well-defined and reproducible relationship. In real road networks, however, complete homogeneity is rarely found, and network-wide congestion is not always apparent. In the case where only a few roads show a different behavior than all the other roads, the observed flow-density relationship lies slightly below the upper bound (11). Otherwise, the empirical MFDs exhibit scatter that leads to a range of observed flows for any given accumulation, which leads to uncertainty in the estimation of the descriptive parameters $(12-15)$. Seminal studies have identified the inhomogeneous spatial distribution of vehicle densities as the reason for this scatter (16, 11, 17-19). A partial solution, in case of contiguous and homogeneous sub-regions, is partitioning $(20,21)$.

This uncertainty in the estimation of the upper bound under real-world traffic conditions and urban dynamics, might result in an inaccurate estimation of the MFD parameters - especially if congestion is rarely observed network-wide (22). For example, if the observed data does not exhibit a distinct congested branch, it is hard to determine the network capacity and critical density. Similarly, as the data normally comes from punctual observations out of the whole network, it is unclear how representative these observations might be (i.e. how much is the observed capacity affected by the network's inhomogeneity). This, in turn, also leads to uncertainties and errors in the parametrization of the MFD for applications (e.g. traffic control), reducing the effectiveness of such applications. This leads to the question addressed in this paper: Given day-to-day fluctuations from limited and probably biased empirical data, how can the shape of the MFD, and especially the critical density for traffic control purposes, as well as the level of inhomogeneity, be accurately estimated?

This paper proposes a novel re-sampling methodology to estimate the level of inhomogeneity and the critical density in urban networks under uncertain traffic conditions, even when only limited empirical data is available. We provide empirical evidence on the performance of the proposed methodology using two large data sets for the cities of Lucerne, Switzerland (1 year) and London, UK (3 weeks). The results show that the proposed methodology estimates the 
critical density accurately for Lucerne, even when no congested branch of the MFD is available. Additionally, we illustrate how the level of inhomogeneity is lower in Lucerne than in the three areas of the network of London that we investigate. Moreover, we show that in the case of London, the capacity of the system could be increased by around $20 \%$, under the assumption that all links behave in a similar manner as the $50 \%$ best links. All things considered, this new methodology promotes the concept and the use of the MFD in real world applications.

The remainder of this paper is organized as follows. The next section introduces the resampling approach, then we present and discuss the two available data sets in detail. Thereafter we show the empirical results. Conclusions are given at the end.

\section{METHODOLOGY}

\section{Re-sampling the MFD}

This section introduces the re-sampling method, which later will be used to estimate the critical density and the level of inhomogeneity in an urban network. The idea of the re-sampling method is to identify the most homogeneous sub-samples of all roads by first creating many random sub-samples of the network, estimating for each an MFD, and extracting the smooth upper bound from the superposition of all MFDs. When the re-sampling parameters are chosen appropriately, all points on the upper bound represent the most homogeneous traffic states.

As an illustration, consider an urban road network in and around a central business district (CBD) with many roads and vehicles. During the morning commute, most roads are congested, but not all of them, e.g. arterial roads leading into the CBD are more congested than those leading out. The resulting averages provide an estimate on the actual mean performance of the road network, but not the potential performance if all links were similarly congested. Choosing a certain sample size and repeating the sampling many times enables to find a homogeneous set of roads without the need to filter the most homogeneous links manually or apply a more complex partitioning (which usually assumes spatial contiguity of the partitioned regions).

The urban road network of a city is given by $N$ directed links, where $N$ refers to all the monitored links (i.e. $N$ might be any number covering between 0 and $100 \%$ of the links in the network). The length of each link $i$ is known as $l_{i}$. Following Geroliminis and Daganzo (12), the MFD is given by the length-weighted means of flow $q$, and density $k, q=\sum_{i} q_{i} l_{i} / \sum_{i} l_{i}$ and $k=\sum_{i} k_{i} l_{i} / \sum_{i} l_{i}$, respectively. As previously discussed, under real conditions a network might not be homogeneously congested, and some links might be more congested than others. If these form a connected subgraph in the road network, we could partition the network as for example in (17). However, if these links are randomly distributed across the network and the sample varies with time, we can estimate the MFD as follows in order to reduce the influence of inhomogeneity in the measurements. We randomly sample $\Omega$-times without replacement $N_{s} \subset N$, where the ratio $N_{s} / N$ denotes the sample size as a proportion of all observations. The maximum number of combinations is given by the binomial coefficient $C=\left(\begin{array}{c}N \\ N_{s}\end{array}\right)$. Notice that sometimes, the number of possible combinations that can be considered might be limited by the computational resources, leading to $\Omega \ll C$.

The intuition behind this statistical sampling is simple. In the case of a network with homogeneous roads (i.e. all exhibiting identical fundamental diagrams) and a homogeneous distribution of congestion, the proof of the equivalence between the upper bound of the full sample and the re-sampled upper bound is trivial. For a more realistic and therefore inhomogeneous network, 
a representative sub-sample with $N_{s} \gg 0$ links will never exceed the theoretical upper bound by Daganzo and Geroliminis (1); the sub-sample will be equal to the upper bound in case of perfect homogeneous congestion, and below the upper bound in all other cases. In other words, estimating average flows and densities for all combinations $C$ (or a subset of $\Omega$ combinations) of a representative subsets of links, increases the chance of obtaining for some combinations and time intervals a homogeneous distribution of congestion, leading to an MFD estimate that is less susceptible to inhomogeneities.

\section{Identifying the critical density and capacity for empirical MFDs}

9 Since the proposed sampling method is repeated $\Omega$ times (i.e. we take $\Omega$ sub-samples of the - same sample size), it significantly increases the scatter and range of flow-density relationships, but exhibits a smooth upper bound. To identify such a stable upper bound for the re-sampled MFD, we use the median of the top $M$ flow values per density bin, where $M$ depends on the number of observations in each bin. We chose the median to avoid any bias due to outliers. After a number of empirical trials, we have defined for most cases $M=50$, but it can decrease down to 5 when the number of observations is small. From this upper bound we define the capacity as the 97.5th percentile of flow to avoid also the influence of outliers. The critical density is then the mean density corresponding to this capacity.

\section{Estimating the level of inhomogeneity}

Following the re-sampling methodology, we propose a measure to estimate inhomogeneity in a network based on the observed capacities in the re-sampled MFD without explicitly considering spatial and temporal effects. The idea is to obtain the highest capacity from all investigated combinations in each sample size, and calculate the relative difference in reference to the observed capacity of the full sample. We define this relative difference as the additional capacity that the network could handle if all links were to behave similarly to the best sub-sample of the respective sample size.

As an illustration, imagine a perfectly homogeneous road network, where all roads carry the same level of traffic. It is clear that for such a case the additional capacity will be zero for all sample sizes. In all other cases, where roads and traffic states are inhomogeneous, we will observe non-zero additional capacities, as we expect that some combinations from lower sample sizes will exclude the constraining roads from the sample. In other words, we estimate the additional capacity as a function of the sample size, and propose to calculate the area below this function - the larger this area is, the less homogeneous the urban network is based on its currently observed state. Notice that the level of inhomogeneity, as defined here, is a measure which is relative to the reference case.

\section{DATA}

This section presents the available data sets for the cities of Lucerne, Switzerland and London, UK. Both cities differ significantly in size, population, and network topology. Lucerne has around 80'000 inhabitants, whereas London has around 100 times as many, with a population density which is around twice as large. The traffic authorities of Lucerne and London operate an extensive network of loop detectors in their cities. Such loop detectors are mainly installed for traffic control and congestion identification purposes. They measure traffic flow (number of vehicles passing the detector) and occupancy (fraction of time the detector is occupied by a vehicle) during a certain observation interval $T$. Due to the system design, the loop detectors 
$$
2
$$

in London are located more frequently at the upstream end of the link, while in Lucerne the detectors are located further downstream. For London, we use three different regions, one around Whitechapel (253 loop detectors), one around Fulham (93 loop detectors), and one around Chelsea (102 loop detectors). For Lucerne, we consider the entire downtown area (158 loop detectors). Figure 1 provides an overview of the two cities, and Table 1 provides some relevant statistics. In the following, we discuss in more detail how the data sets were prepared.

In order to locate each detector, we geo-referenced all spatial information of the loop detector positions in reference to the whole road network. Connecting the network and the loops has several advantages. First, MFDs for arbitrary shaped perimeters can be estimated, and potential partitioning can be carried out. Second, attributes of the road network, e.g. road type and speed limit, can be linked to the loop detectors, allowing further filtering, e.g. removing residential roads (14) that generally have no connecting character. Third, we enriched the data with information on the driving direction of each lane covered by a loop detector. This allowed us to identify multiple detectors per lane, and remove some to avoid any duplicity. For this analysis, we queried the road network from OpenStreetMap. At the end, we selected for this study only loops located on trunk, primary, secondary, and tertiary roads.

It is clear that empirical datasets are error prone. Both Lucerne and London have an internal system that detects malfunctioning loops. Nevertheless, we defined a set of rules, whose objective is to identify potentially malfunctioning loop detectors as in (23). We automatically identified measurements as false when no variation of the values were registered during a full day, $80 \%$ of the values were zero, either flow or occupancy were zero while the other was not (as long as the occupancy was smaller than 0.95 ), and obviously when the internal system itself reported an error. Additionally, we inspected each loop detector's scatter plot and verified the results of the filtering. The error rate is 30\% (23\% internal system, $7 \%$ verification) for Lucerne and $31 \%$ (30\% internal system, $1 \%$ verification) for London.

\section{Occupancy-density conversion}

Loop detectors measure flow and occupancy. Traffic density can be approximated from occupancy using a scalar conversion $(24,25,23)$. The scalar corresponds to the mean vehicle length as seen by the detector, i.e. the sum of vehicle length and detector length (26). Hall and Persaud (25) and Coifman (26) showed that this scalar conversion is a good approximation with small errors in uncongested traffic. Even though previous research predominantly focused on highways, based on recent findings we assume that this scalar conversion also holds for urban networks (14). In the context of MFD research, values around $0.005 \mathrm{~km}$ have been identified for the mean vehicle length: $0.0053 \mathrm{~km}$ for Yokohama (12) and $0.0063 \mathrm{~km}$ for Zurich (14). We estimate the mean effective vehicle length in Lucerne to be $0.0063 \mathrm{~km}$ and for London to be $0.006 \mathrm{~km}$.

\section{RESULTS}

In this section, we present the results of the re-sampling methodology using the data described above.

\section{Empirical MFDs}

First, let us show the full-sample MFD for both cities; for London we choose Whitechapel as an example. Figure 2 displays the MFD for 258 working days (i.e. one full year) for the city 


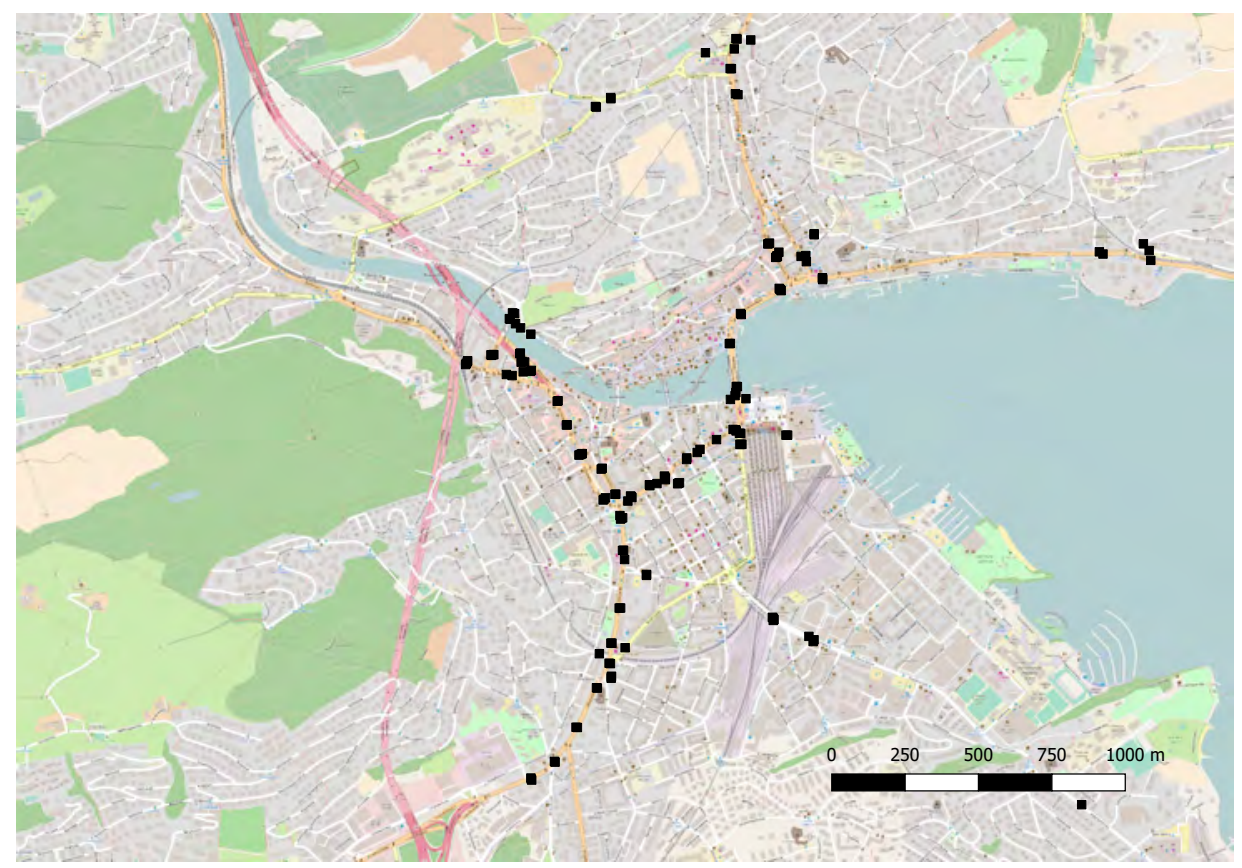

(a) Lucerne.

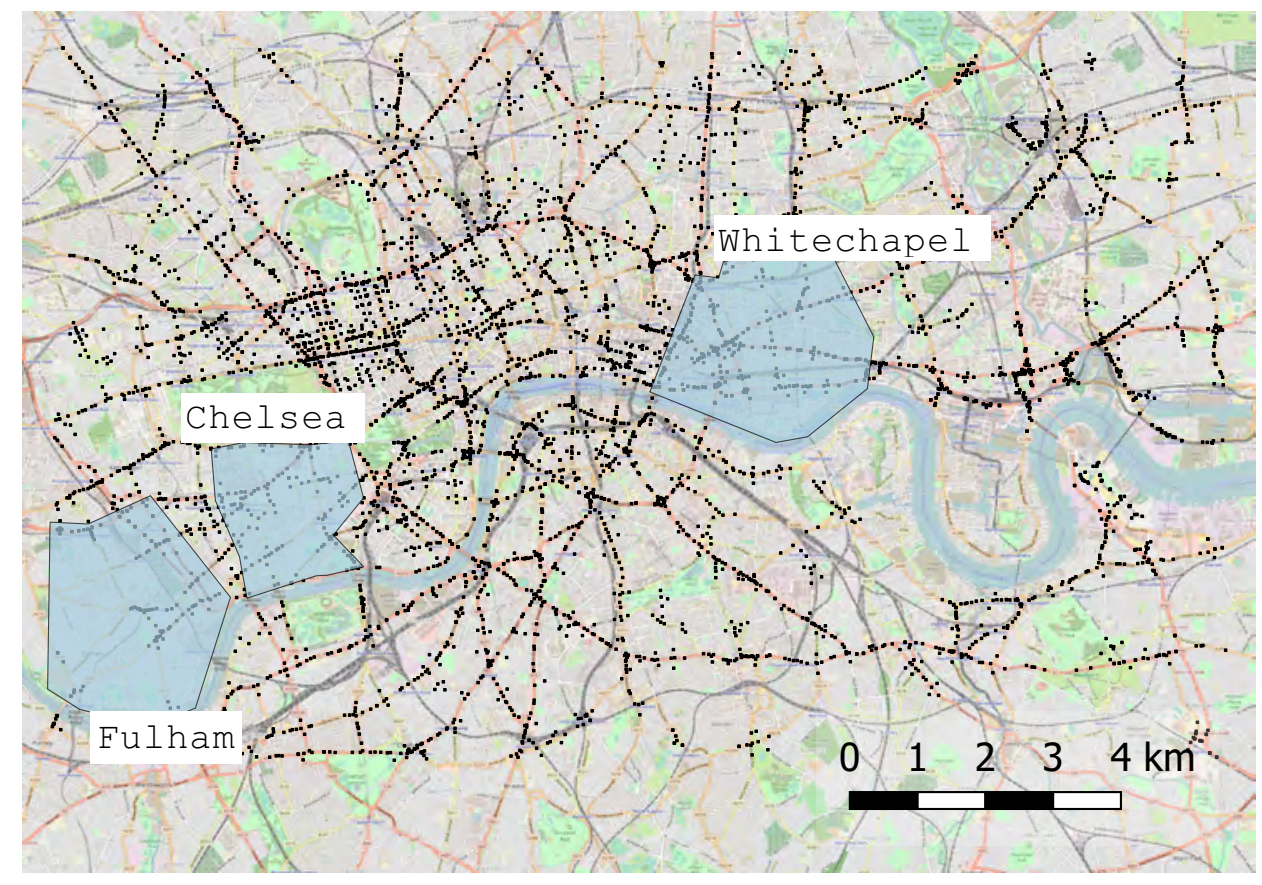

(b) London.

FIGURE 1 Regions in Lucerne and London which are analyzed. Loop detectors are represented as black squares. Both maps are oriented towards north. 
TABLE 1 Overview of the two cities and the respective data set.

\begin{tabular}{|c|c|c|c|}
\hline City & & Lucerne $(\mathrm{CH})$ & London (UK) \\
\hline Total population & & $81^{\prime} 000$ & $8^{\prime} 500^{\prime} 000$ \\
\hline Total number of detectors & & 158 & $5^{\prime} 719$ \\
\hline Total lane-km covered & {$[\mathrm{km}]$} & 26 & 1'298 \\
\hline Aggregation interval & [min] & 3 & 5 \\
\hline Number of working days & & 258 & 15 \\
\hline
\end{tabular}

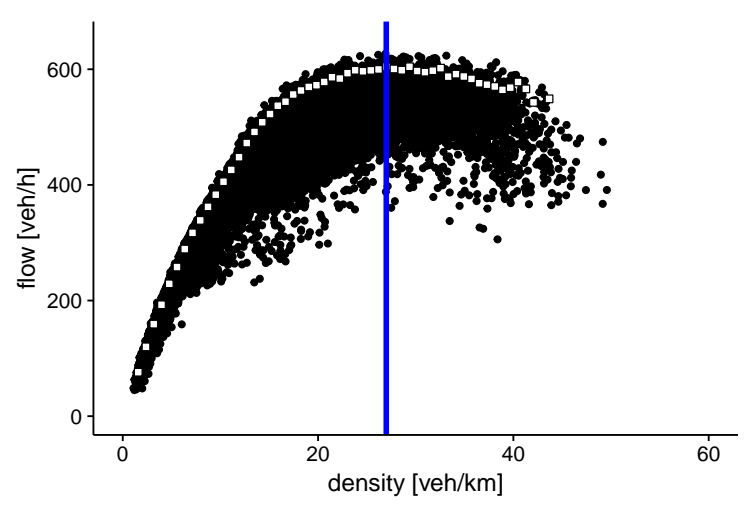

(a) Lucerne

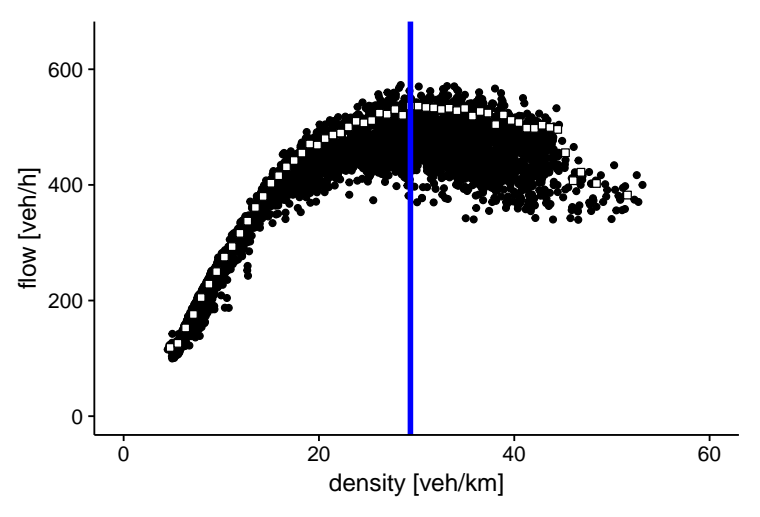

(b) Whitechapel, London

FIGURE 2 MFDs from all days in which data is available and the upper bound. Estimated critical density is indicated by the vertical line.

of Lucerne, and 15 working days for Whitechapel, both in the respective aggregation interval (see Table 1). Every point represents a macroscopic traffic state in terms of vehicle density and vehicle flow for every aggregation interval during the observation period, indicated in Table 1. Both regions show an uncongested branch and the beginning of the congested branch. It is not surprising that Lucerne shows a higher level of scatter, as there are significantly more days included in the dataset. We have added the stable upper bound, as defined previously, in white squares, as well as a line indicating the critical density. The capacity and the average speed in the uncongested branch of Lucerne's MFD are slightly higher than those in London, whereas the critical density is slightly lower. We attribute this to the difference in the network topology and traffic control. More details can be found in (27).

Remarkably, both MFDs exhibit a smooth upper bound, which supports the general theory of the MFD, defined as a tight upper bound relatively independent of demand. Notice also that, to the best of our knowledge, this is the first time that a full year MFD is estimated. It shows relatively little variation which indicates that empirical MFDs can indeed be used for control schemes and other applications demanding a long-term invariance.

\section{Estimating the critical density}

7 The few existing MFD empirical studies show that in reality, data availability is very often s limited and only few days can be used for analysis. Empirical studies rely on observation 19 periods that usually range between 1 and 6 days, which show little or no congestion making an 
estimation of the critical density hard or even impossible (e.g. 12, 13, 28, 14). In the following, we investigate how the proposed re-sampling method performs in the estimation of the critical density when only limited data is available, e.g. if we have only data from 1 day, 3 consecutive days, or 6 consecutive days, and not all exhibit a distinct congested branch. We focus on the city of Lucerne, and use the previously shown upper bound of the 1-year MFD of Lucerne as the reference MFD (see Figure 2(a)).

For the re-sampled MFDs we choose a total of 4 sample sizes $(20 \%, 40 \%, 60 \%$ and $80 \%$ of the full sample), and the number of draws without replacement $\Omega$ is set to 1'000. For a sensitivity analysis we also vary the observation period for each MFD estimation using either 1 , 3 , or 6 consecutive day(s). We then try to determine the critical density for each of the estimated MFDs. As an example, we randomly select $20 \%$ of Lucerne's loop detectors, create the MFDs thereof, and repeat this 1'000 times re-selecting another $20 \%$ of loop detectors each time for every set of 1, 3 and 6 consecutive day(s). Notice that not every MFD shows a decreasing branch, thus it is not possible to properly determine a critical density for every estimated MFD. We assume that the determined value of the critical density is valid only when the MFD shows a decrease in flow of at least $30 \mathrm{veh} / \mathrm{h}$ (around $5 \%$ of the capacity in the observed cases) for densities higher than the determined value of the critical density. Figure 3 shows the results of the critical density estimation for the MFD of Lucerne. Figure 3(a) shows the percentage of successful critical density estimations, for each combination of sample size and observation period. In case of an observation period of 1 day, the maximum number of estimations of critical densities is 258, in case of 3 days that number is 86 , and in case of 6 days the number is 43 . From the figure we first see that as we reduce the sample size, the percentage of days where we can properly determine the critical density increases significantly. As a matter of fact, we can effectively determine the critical density $100 \%$ of the time when the sample size is $40 \%$ or lower and the number of available days is 3 or 6 . In addition, and not surprisingly, the more days we include in the estimation of the MFD, the more likely we are to observe a valid critical density. For example, if we had only 1 day of data, then an estimation of the critical density would only be possible during $6 \%$ of all days (14 days) using the full sample (100\% sample size), whereas if we had 3 days of available data, we could estimate the critical density in $19 \%$ of all cases. These results show how our re-sampling methodology can effectively increase our ability to determine the critical density, even when only limited data is available, e.g. when no clearly congested branch is apparent in the MFD.

Given that it is possible to determine a critical density for a given day, Figure 3 (b) shows the range of the relative errors of such densities compared to the reference critical density, identified from the 1-year MFD. For brevity, we only show the results for the 1 day estimates; the results for the 3 and 6 days look similar. In the figure, an overestimation of the critical density has a positive sign. Interestingly, the accuracy remains within a similar range for all sample sizes (the different sample sizes include the number of observations given in Figure 3(a)). In other words, even as we decrease the sample size to increase the number of days where we can determine the critical density, the error in the estimation remains approximately the same. As a matter of fact, we can see that for the lowest two sample sizes (20\% and $40 \%)$ we only underestimate the value of the critical density by less than $2 \%$ in the median. To conclude, this clearly indicates that the re-sampling method does not only increase our ability to estimate the critical density, but also maintains the level of accuracy of such estimation.

As a further validation, we compared the error distributions from the full sample and the re-sampling method using a Kolmogorow-Smirnow test. We found a high p-value (0.4) for all sample sizes indicating that they all come from the same distribution of errors. We refrain from 


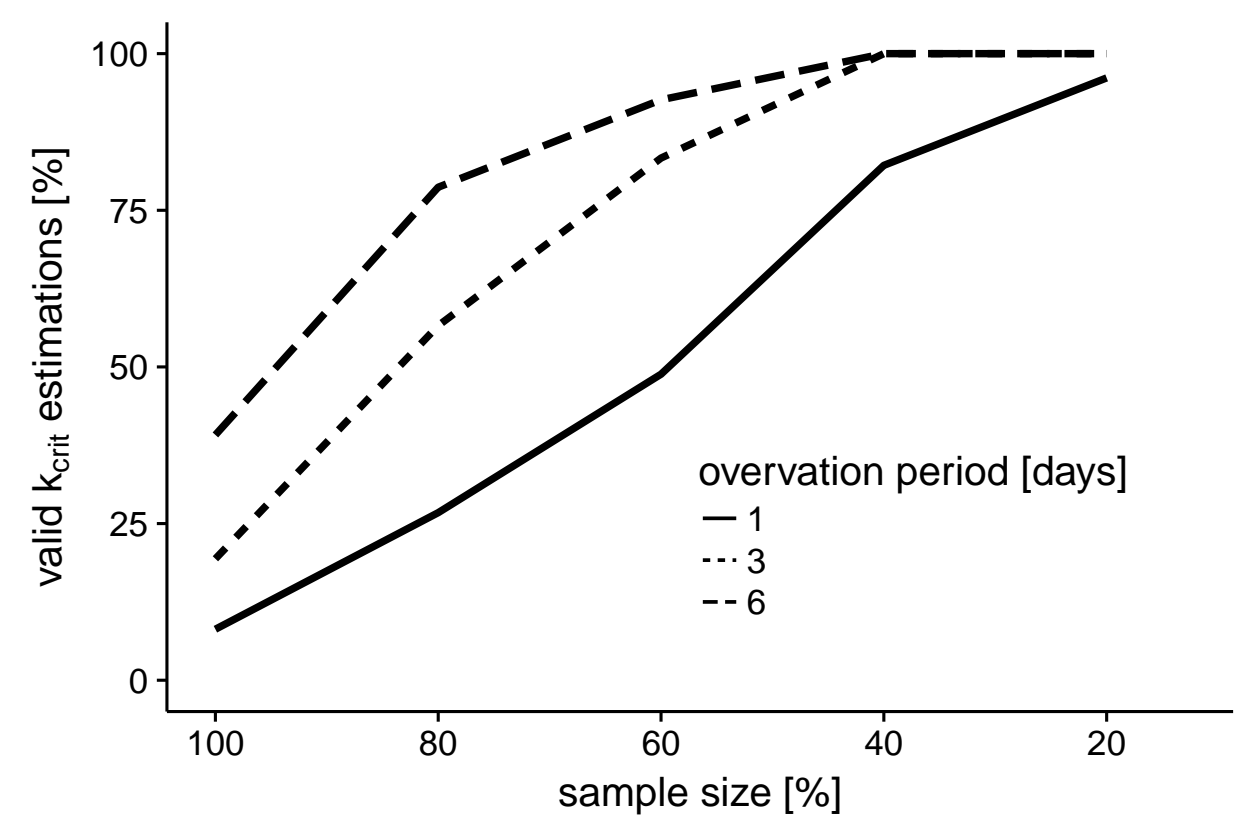

(a) Number of valid critical density estimations.

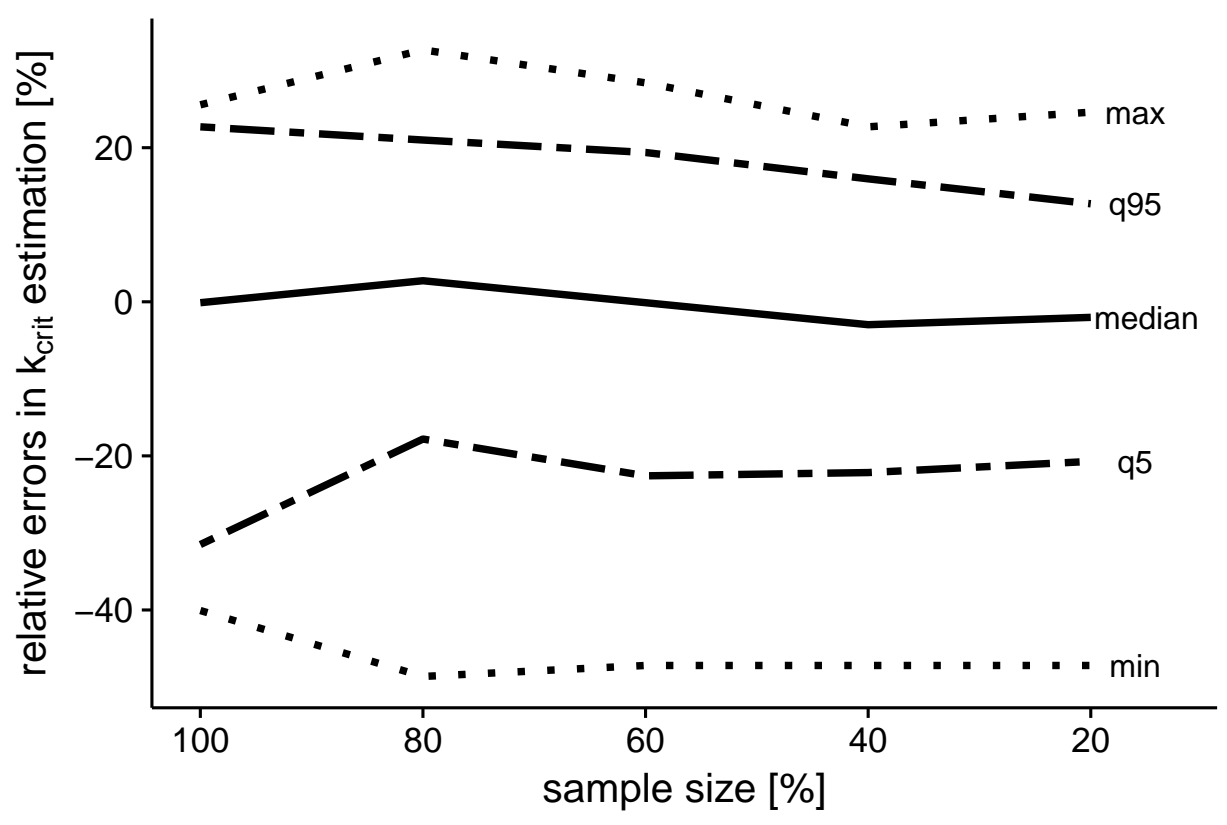

(b) Errors in the critical density estimation using the re-sampling methodology for an observation period of 1 day.

\section{FIGURE 3 Results for the critical density estimation.}

1 showing the same results for London, as for that network, every day exhibits a congested branch of the MFD. Hence, there is no need to apply our method for identifying the critical density.

\section{Estimating the level of inhomogeneity}

4 We will now discuss the second application of our re-sampling method. The experimental set-up 5 is similar to the previous section, but now we focus on the capacity instead of the critical density.

6 Figure 4 shows our approach to measure the level of inhomogeneity in a city. We show the 
relative capacity increase as a function of the sample size for Lucerne and the 3 different regions in London. As expected, the lower the sample size, the higher the relative difference is. We attribute this to the fact that with smaller sample sizes, it is easier to identify combinations of roads that exhibit a behavior exceeding the average in terms of vehicle flow. Two issues, however, deserve some consideration: First, it is clear that a certain number of roads need to be included in order to have a representative and meaningful sub-sample for the investigated network. We set the minimal sample size to $10 \%$. Second, the increases in capacity are of theoretical nature, because they would only be observed if all roads were to behave like the best roads in the sub-sample. For example, we see that the region around Whitechapel in London would have a $19 \%$ higher capacity if all roads were to behave like the $50 \%$ best roads.

Regarding the differences between the different regions in Figure 4 , we notice that Lucerne has the lowest curve and therefore the smallest area below the curve (i.e. integral of the curve) compared to the three regions in London. Thus, we conclude that Lucerne exhibits the least amount of inhomogeneity from the investigated sample. We attribute this to (i) the size of the network, which offers only a few higher level roads, and (ii) to the limited feasible route alternatives that are possible in Lucerne in combination with the very limited number of ODpairs within this small city. Within the investigated perimeter, the south and the north of the city are only connected by two bridges (see Figure 1(a)); all other bridges are pedestrian bridges. In other words, for the few OD combinations that exist, there are only 1 or 2 routes available, constraining route choice. Conversely, for the regions of the city of London, we see from the map in Figure 1(b) that the network is connected with more higher level roads and thus, the number of feasible routes is larger. In other words, many route options combined with many OD-pairs increases the likelihood of an inhomogeneous distribution of traffic. When we calculate the area below the curves as an indicator for the level of inhomogeneity, we find that the areas in London are around 1.5 to 2 times larger than the one in Lucerne. Interestingly, the three curves for the London area follow the same trend, including a relatively sharp increase at the full sample size (100\% sample size). This indicates that a few roads exist in the sample, which have a significant impact on the average capacity of the network. In the best case, removing the $10 \%$ worst roads (in terms of capacity) from the sample, theoretically increases the network capacity by around $15 \%$.

Notice that this approach explicitly quantifies the level of inhomogeneity from the shape of the MFD, without considering spatial nor temporal variations in traffic volumes and densities. However, the method does have some clear advantages: it is easy to use, requires very little inputs, and is robust to the placement bias of loop detectors (13;14). Link-based partitioning methods might include such a bias if they are based on traffic densities. For the re-sampling method, on the other hand, we consider the spatial mean of the densities, as multiple detectors are included in every sample. Hence, this method represents the first of its kind for assessing and identifying the level of inhomogeneity in an urban road network.

\section{DISCUSSION AND CONCLUSIONS}

In this paper we introduced a novel methodology that allows us to estimate the critical density of the MFD, even when no congested branch is observed, and to estimate the level of inhomogeneity within the network. The proposed methodology is based on the idea of re-sampling the empirical data set. Using an extensive data set, which included the cities of Lucerne, Switzerland, (1 year) and London, UK, (3 weeks) we provided insights on the performance and the application of the re-sampling method. First, we estimated the critical density of the MFD using limited data and found that we can drastically increase the number of days on which an estimation is possible - 


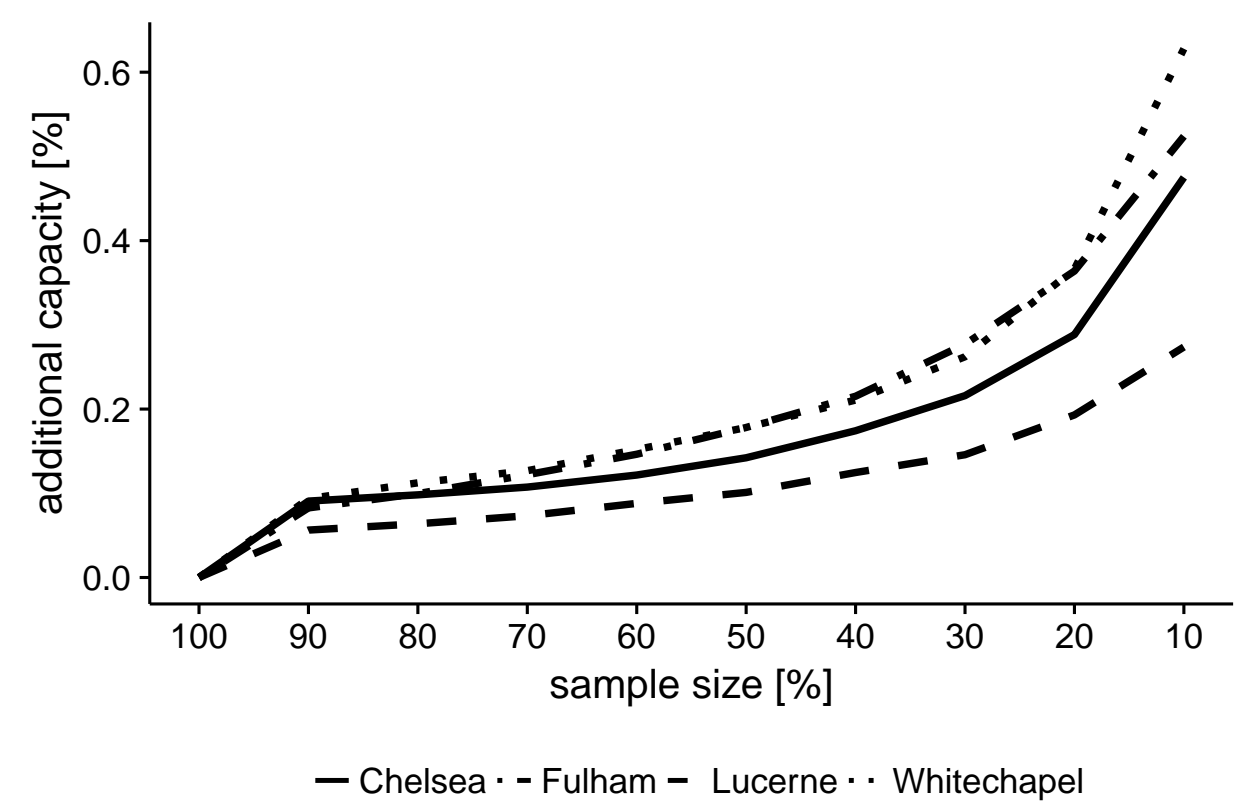

\section{FIGURE 4 Results of the analysis on the level of inhomogeneity. All results cover a period of 15 consecutive weekdays.}

even if the network does not show network-wide congestion every day. This is important, as very often, access is only given to data of a very limited time period. We showed that for the network of Lucerne, we can increase the fraction of days that allow an estimation of the critical density, based on data of 1 day, from $6 \%$ to $94 \%$ while keeping a similar level of accuracy. Such robust estimation of the empirical critical density is crucial for many control algorithms. Second, using the re-sampling method, we quantified the level of inhomogeneity in urban networks. For London, we find that the capacity of the system could be increased by around $20 \%$, under the assumption that all links behave in a similar manner as the $50 \%$ best links. For Lucerne this value is around $10 \%$. We propose to estimate the level of inhomogeneity as the area below the curve relating the sample size and the capacity increase. In summary, the re-sampling method presented in this paper is very promising for different application purposes of the MFD. It is easy to use, requires only very few inputs, and is robust against a potential placement bias in loop detector data.

There have been numerous studies investigating the performance of the network when implementing a perimeter control scheme based on the MFD. However, the estimation of the required critical density has not yet been discussed in an empirical context and only few of the cities investigated up to now, exhibit a strong congested branch. The re-sampling methodology proposed in this paper provides a promising approach to estimate the critical density empirically at large urban scale.

Additionally, the proposed measure of the level of inhomogeneity gives city planners the possibility to analyze and quantify how efficiently their road network is utilized. This line of questions was started over 50 years ago by Smeed (29), but can now be evaluated in more detail with the availability of big data.

Future research will concentrate on how the re-sampling method and the theoretical upper bound from analytical approaches relate to each other. In other words, it should address the question of whether we can approach the theoretical upper bound with our re-sampling 
methodology. Furthermore, additional insights could be gained from an application of the methodology to floating car data. On-going studies are also evaluating the possibility to estimate an infrastructure potential using the MFD, and thereby deepen the understanding of the effects of inhomogeneous traffic in urban networks.

\section{ACKNOWLEDGMENTS}

This work was supported by ETH Research Grants ETH-04 15-1 and ETH-27 16-1. We especially thank Thomas Karrer and Milena Scherer from the city of Lucerne and Ashley Turner and Andy Emmonds from Transport for London (TfL) for their collaboration.

\section{REFERENCES}

1. Daganzo, C. F. and N. Geroliminis (2008) An analytical approximation for the macroscopic fundamental diagram of urban traffic, Transportation Research Part B: Methodological, 42 (9) 771-781.

2. Aboudolas, K. and N. Geroliminis (2013) Perimeter and boundary flow control in multireservoir heterogeneous networks, Transportation Research Part B: Methodological, 55, $265-281$.

3. Haddad, J. and N. Geroliminis (2012) On the stability of traffic perimeter control in tworegion urban cities, Transportation Research Part B: Methodological, 46 (9) 1159-1176.

4. Geroliminis, N. and D. M. Levinson (2009) Cordon pricing consistent with the physics of overcrowding, in Transportation and Traffic Theory 2009: Golden Jubilee, 219-240.

5. Zheng, N., R. A. Waraich, N. Geroliminis and K. W. Axhausen (2012) A dynamic cordon pricing scheme combining a macroscopic and an agent-based traffic model, Transportation Research A, 46 (8) 1291-1303.

6. Zheng, N., G. Rérat and N. Geroliminis (2016) Time-dependent area-based pricing for multimodal systems with heterogeneous users in an agent-based environment, Transportation Research Part C: Emerging Technologies, 62, 133-148.

7. Boyaci, B. and N. Geroliminis (2011) Estimation of the network capacity for multimodal urban systems, Procedia - Social and Behavioral Sciences, 16, 803 - 813.

8. Zheng, N. and N. Geroliminis (2013) On the distribution of urban road space for multimodal congested networks, Transportation Research Part B: Methodological, 57, 326 - 341.

9. Schreiber, A., A. Loder and K. W. Axhausen (2016) Urban mode and subscription choice An application of the three-dimensional MFD, paper presented at the 16th Swiss Transport Research Conference, Ascona, May 2016.

10. Leclercq, L., N. Chiabaut and B. B. Trinquier (2014) Macroscopic Fundamental Diagrams: A cross-comparison of estimation methods, Transportation Research Part B: Methodological, 62, 1-12.

11. Knoop, V. L., H. van Lint and S. P. Hoogendoorn (2015) Traffic dynamics: Its impact on the Macroscopic Fundamental Diagram, Physica A: Statistical Mechanics and its Applications, 438, 236-250. 
12. Geroliminis, N. and C. F. Daganzo (2008) Existence of urban-scale macroscopic fundamental diagrams: Some experimental findings, Transportation Research Part B: Methodological, 42 (9) 759-770.

13. Buisson, C. and C. Ladier (2009) Exploring the impact of homogeneity of traffic measurements on the existence of macroscopic fundamental diagrams, Transportation Research Record: Journal of the Transportation Research Board, 2124, 127-136.

14. Ambühl, L., A. Loder, M. Menendez and K. W. Axhausen (2017) Empirical Macroscopic Fundamental Diagrams: New Insights from Loop Detector and Floating Car Data, Presented at 96th Annual Meeting of the Transportation Research Board.

15. Muhlich, N., V. V. Gayah and M. Menendez (2015) An Examination of MFD Hysteresis Patterns for Hierarchical Urban Street Networks Using Micro-Simulation, Transportation Research Record: Journal of the Transportation Research Board, 2491, 117-126.

16. Geroliminis, N. and J. Sun (2011) Properties of a well-defined macroscopic fundamental diagram for urban traffic, Transportation Research Part B: Methodological, 45 (3) 605-617.

17. Ji, Y. and N. Geroliminis (2012) On the spatial partitioning of urban transportation networks, Transportation Research Part B: Methodological, 46 (10) 1639-1656.

18. Gayah, V. V. and C. F. Daganzo (2011) Clockwise hysteresis loops in the macroscopic fundamental diagram: An effect of network instability, Transportation Research Part B: Methodological, 45 (4) 643 - 655.

19. Daganzo, C. F., V. V. Gayah and E. J. Gonzales (2011) Macroscopic relations of urban traffic variables: Bifurcations, multivaluedness and instability, Transportation Research Part B: Methodological, 45 (1) 278 - 288.

20. Ji, Y., J. Luo and N. Geroliminis (2014) Empirical observations of congestion propagation and dynamic partitioning with probe data for large-scale systems, Transportation Research Record: Journal of the Transportation Research Board, 2422, 1-11.

21. Saeedmanesh, M. and N. Geroliminis (2017) Dynamic clustering and propagation of congestion in heterogeneously congested urban traffic networks, Transportation Research Procedia, 23 (00) 962-979.

22. Gao, X. and V. V. Gayah (2017) An analytical framework to model uncertainty in urban network dynamics using macroscopic fundamental diagrams, Transportation Research Procedia, 23, 497-516.

23. Bickel, P., C. Chen, J. Kwon, J. Rice, E. v. Zwet and P. Varaiya (2007) Measuring traffic, Statistical Science, 22 (4) 581-597.

24. Athol, P. (1965) Interdependence of certain operational characteristics within a moving traffic stream, in Highway Research Record 72, 58-87, National Research Council, Washington, D.C.

25. Hall, F. L. and B. N. Persaud (1989) Evaluation of speed estimates made with single-detector data from freeway traffic management systems, Transportation Research Record: Journal of the Transportation Research Board, 1232, 9-15. 
26. Coifman, B. (2001) Improved velocity estimation using single loop detectors, Transportation Research Part A: Policy and Practice, 35, 863-880.

27. Loder, A., L. Ambühl, M. Menendez and K. W. Axhausen (2017) Empirics of multi-modal traffic networks - Using the 3D macroscopic fundamental diagram, Transportation Research Part C: Emerging Technologies, 82, 88-101.

28. Jiyang, B., H. van Zuylen and L. Shoufeng (2016) Determining the Macroscopic Fundamental Diagram on the Basis of Mixed and Incomplete Traffic Data, Presented at the 95th Annual Meeting of the Transportation Research Board, 1-13.

8 29. Smeed, R. J. (1961) The traffic problem in towns, Statistical Society, Manchester. 